\title{
DC Magnetic Properties of Amorphous Vitrovac Ribbon
}

\author{
P. KOlláR ${ }^{a, *}$, Z. BirČÁKOVÁ ${ }^{a}$, J. FÜZER $^{a}$ AND M. KuŹMińSKI ${ }^{b}$ \\ ${ }^{a}$ Institute of Physics, Faculty of Sciences, P.J. Šafárik University, Park Angelinum 9, 04154 Košice, Slovakia \\ ${ }^{b}$ Institute of Physics, Polish Academy of Sciences, Aleja Lotnikow 32/46, PL-02668 Warsaw, Poland
}

\begin{abstract}
Soft magnetic amorphous Co-based materials prepared by rapid quenching method in the form of thin ribbon are well-known due to their excellent soft magnetic properties as high permeability, low coercivity, and low magnetic losses in $\mathrm{kHz}$ range. The amorphous $\mathrm{Co}-\mathrm{Fe}-\mathrm{B}-\mathrm{Si}$ material Vitrovac(R) 6155 U55 produced by Vacuumschmelze $\mathrm{GmbH} \& \mathrm{Co}$. KG belongs to this class of materials and was investigated in as-delivered state. The aim of this work was to study DC magnetization process by various experimental methods. We have measured magnetization curve by fluxmeter based hysteresis graph and hysteresis loops by three different fluxmeter based hysteresis graphs, exhibiting significant differences. The first and the second hysteresis graph perform the hysteresis loops measurement by the point-by-point method, either with commutative or with summing steps. The third one is AC hysteresis graph working at very low frequencies down to $7 \mathrm{mHz}$, performing the continuous method. The explanation of this result is based on the structural after effect influencing the domain wall displacement. We assumed that the domain structure consists of very small number of domain walls responsible for magnetization process, which was confirmed by the visualization of static domain structure by a computer-controlled setup based on the Kerr effect.
\end{abstract}

DOI: 10.12693/APhysPolA.131.675

PACS/topics: 75.60.Jk, 75.60.Ch, 75.60.Ej

\section{Introduction}

Soft magnetic amorphous materials (metallic glasses) prepared by rapid quenching method in the form of thin ribbon are well-known due to their excellent soft magnetic properties as high permeability, high enough saturation induction, and low coercivity. The amorphous Cobased materials exhibit high permeability and low magnetic loss in kHz-range. These properties determine these materials for many applications as signal transformers, chokes, power transformers for the $\mathrm{kHz}$ range and flexible antennae in keyless entry systems [1]. The aim of this work was to study the specificity of DC magnetization process of the low anisotropy amorphous Co-based material.

\section{Experimental}

The amorphous Co-Fe-B-Si material Vitrovac $\mathbb{R} 6155$ U55 F produced by Vacuumschmelze GmbH \& Co. KG was investigated in as-delivered state [2]. The ribbon sample ( $8.2 \mathrm{~m}$ long, $3.8 \mathrm{~mm}$ wide and $21 \mu \mathrm{m}$ thick) for DC magnetic measurements was wound as a core of the toroidal transformer (with diameter of $5.8 \mathrm{~cm}$ ) with primary magnetization coil and secondary pick-up coil.

The magnetization curve was measured by fluxmeter based hysteresis graph and the saturated hysteresis loops by three different fluxmeter based hysteresis graphs. The first hysteresis graph performs the hysteresis loop measurement by the point-by-point (ballistic) method [3], with the commutative steps through the maximum magnetic field values (reference points). This method offers the

\footnotetext{
*corresponding author; e-mail: peter.kollar@upjs.sk
}

possibility for measuring each point of hysteresis loop independently of the previously measured point during one cycle of magnetization reversal. For each point (number of points can be set illimitably) the measuring procedure consists of the magnetic field setting in the following order: positive saturation state, measured point on descending part of hysteresis loop, negative saturation state and measured point on ascending part of hysteresis loop. Magnetic field is fixed in each point for $4 \mathrm{~s}$, then the computer reads the fluxmeter output. The value of magnetic induction of each point of the descending part is calculated as the decrease of the magnetic induction from saturation (similar for ascending part of hysteresis loop). This procedure enables to completely eliminate the drift of the fluxmeter. The second hysteresis graph uses the point-by-point method [3] as well, but with the summing steps through the sequential magnetic field values along hysteresis loop (Permeameter AMH-1K-S Laboratorio Elettrofisico). The third one is the AC fluxmeter based hysteresis graph working at very low magnetization frequencies down to $7 \mathrm{mHz}$ (with triangular waveform), performing the continuous (hysteresis graph) method [3].

The static domain pattern was visualized in the central part of the sample (piece of a ribbon with effective length $8 \mathrm{~cm}$ ) by a computer-controlled set-up based on the Kerr effect with the sensitivity along ribbon axis in various values of DC magnetic field (during magnetization reversal process).

\section{Results and discussion}

The hysteresis loop (selected part) measured up to maximum magnetic field of $20 \mathrm{~A} / \mathrm{m}$ by the point-by-point method with the commutative steps through maximum field values, is shown in Fig. 1. As we can see the descending part of the hysteresis loop does not decrease mono- 
tonously and ascending part does not increase monotonously. We can assume that the domain pattern consists of only few domain walls, the magnetization process is performed unrepeatably in each cycle of magnetization reversal and the domain walls can be pinned on different positions even in the case of the same magnetic field.

In the position of the domain walls during magnetization reversal the processes of stabilization occur, based on structural relaxation associated with the short displacements of atom pairs in the domain wall, leading to the decrease of the domain wall energy [4].

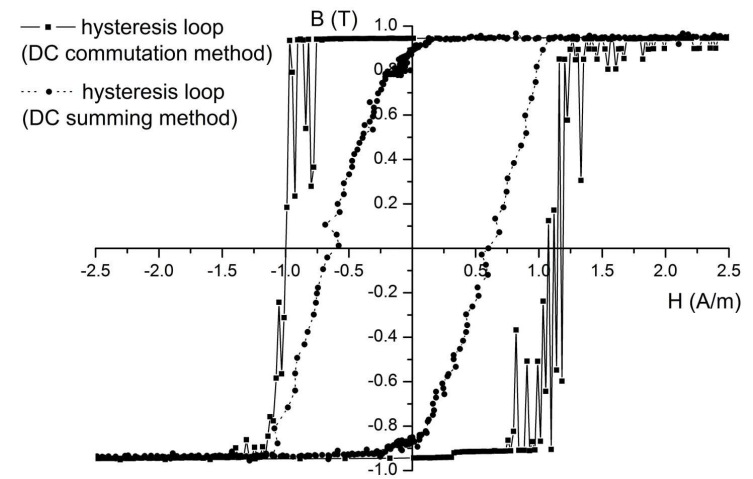

Fig. 1. The DC hysteresis loops of Co-based amorphous ribbon measured by the point-by-point methods, with commutative and summing steps.

Then stronger magnetic field is required there to release the domain wall from local energy minimum. In the saturated state without domain structure the locality of the domain wall position relaxes and the pinning centre of the domain wall vanishes. Due to small anisotropy of the material and high homogeneity, the nuclei of domains with opposite oriented magnetization are created in the largest field, which is why the coercivity is higher than expected.

The measurement of the hysteresis loop performed by the point-by-point method with the step-like summing of flux variations along the loop, consists of two stages. In the first stage during preliminary measurements the function of time dependence of the magnetic field $H(t)$ is created. In the case of low coercive material with nearto-rectangular loop the function $H(t)$ for descending part is not completely decreasing, due to non-zero experimental uncertainty it consists of minority positive steps (the magnetization process is along minor asymmetrical loop at this moment, causing the lowering of the coercivity). This leads to the incorrect low value of the coercivity. The final measurement process (in maximum magnetic field of $20 \mathrm{~A} / \mathrm{m})$ is controlled by the function $H(t)$, but from the point of view of domain walls their displacement is a little bit different leading to the special artefacts mainly at coercivity and remanence, Fig. 1. The ascending part of hysteresis loop has the same defects.

The measurement of AC (with triangle waveform) hysteresis loops with decreasing frequency leads to the quasi $\mathrm{DC}$ loops at the frequency of $7 \mathrm{mHz}$ (the continuous met- hod), Fig. 2. Further decrease of the frequency does not positively influence on the hysteresis loop shape (the total measurement time is longer and the drift of the fluxmeter causes visible defect of the hysteresis loop).

The fact that the magnetization curve measured by the commutation method is inside the hysteresis loop, partially supports the statement that the result of the measuring of the hysteresis loop in AC magnetic field with frequency of $7 \mathrm{mHz}$ is the correct quasi DC loop, Fig. 2 .

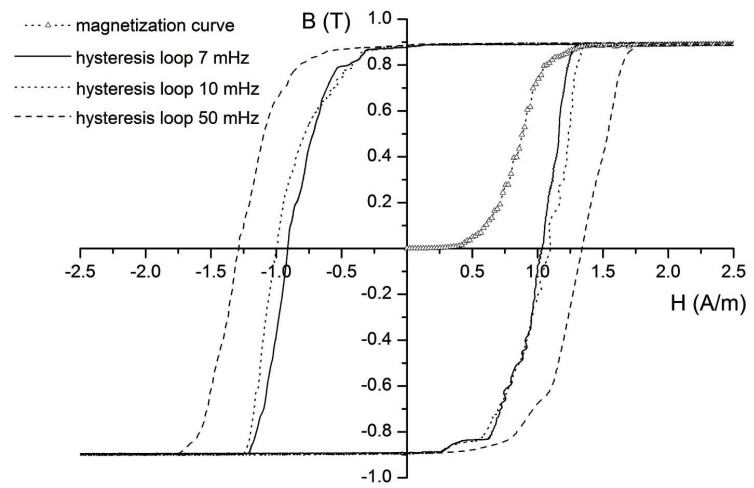

Fig. 2. The AC hysteresis loops measured at different frequencies of the magnetic field with triangle waveform and the magnetization curve measured by point-bypoint method of Co-based amorphous ribbon.

The assumption of the low number of domain walls moved in magnetization reversal process was confirmed by the visualization of the domain structure taken on the shiny side of the ribbon surface, Fig. 3a. Similar domain pattern consisting of three domain walls separating domains with opposite oriented magnetization vectors $\left(180^{\circ}\right.$ domain walls) was also detected on the wheel side of the ribbon, Fig. 3b.
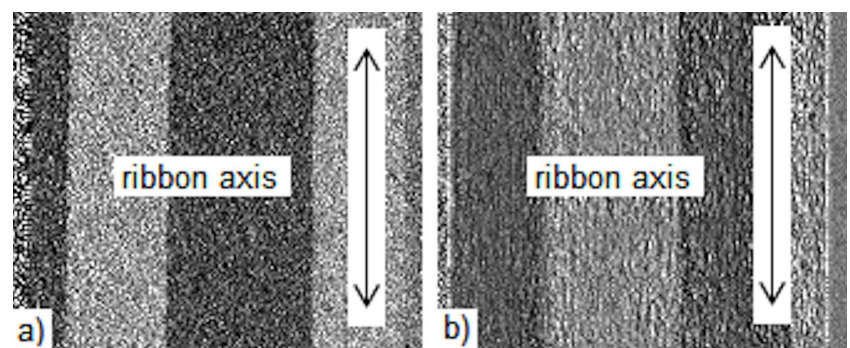

Fig. 3. The domain structure of the Co-based amorphous ribbon: (a) on the shine side of the ribbon, (b) on the wheel side of the ribbon. The sensitivity was oriented along ribbon axis.

The displacement of the domain walls from positive saturation state to negative saturation state can be followed in domain patterns in Fig. 4. The domain structure in Fig. 4a taken in magnetic field of $24 \mathrm{~A} / \mathrm{m}$ (after saturation in magnetic field of $80 \mathrm{~A} / \mathrm{m}$ ) has a relatively long, but narrow domain with opposite oriented magnetization vector in upper-left corner of the picture. The length of the domain increases with the decrease of the 
magnetic field, Fig. 4b (magnetic field reaches the value of $16 \mathrm{~A} / \mathrm{m}$ ). Another long and narrow domain was created probably from lower part of the picture. The volume of the domains with opposite oriented magnetization vector increases with the decrease of the magnetic field, Fig. 4c. Further evolution of the magnetization reversal process to negative saturation can be seen in Fig. $4 \mathrm{~d}-\mathrm{g}$. It is interesting that at the decrease of the magnetic field one of the domain walls remained pinned (domain wall I in Fig. 4d,e) and the second one was moved (domain wall II).

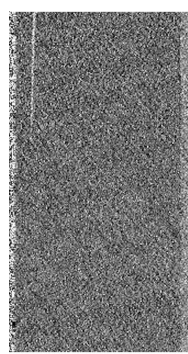

a) $24 \mathrm{~A} / \mathrm{m}$

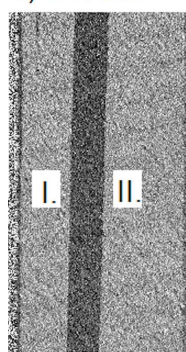

e) $-8 \mathrm{~A} / \mathrm{m}$

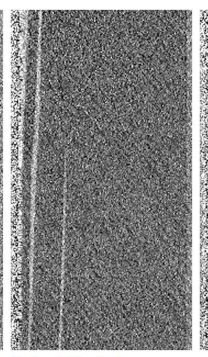

b) $16 \mathrm{~A} / \mathrm{m}$

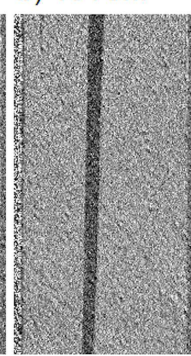

f) $-16 \mathrm{~A} / \mathrm{m}$

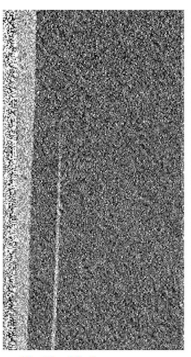

c) $8 \mathrm{~A} / \mathrm{m}$

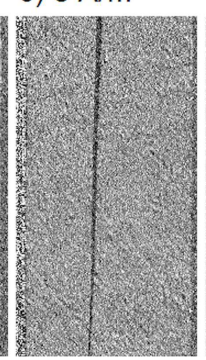

g) $-24 \mathrm{~A} / \mathrm{m}$

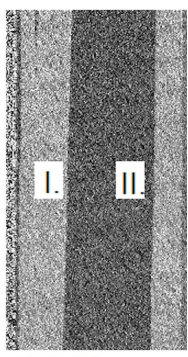

d) $0 \mathrm{~A} / \mathrm{m}$

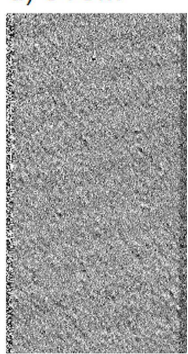

h) $-32 \mathrm{~A} / \mathrm{m}$
Fig. 4. The domain structure of the Co-based amorphous ribbon on the shiny surface taken by Kerr effect (with sensitivity oriented along ribbon axis) from positive saturation to negative saturation. The values of magnetic field are present in the pictures.

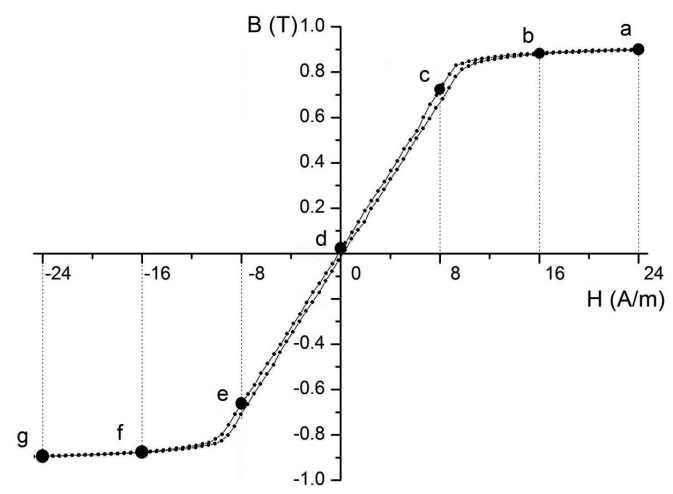

Fig. 5. The quasistatic hysteresis loop measured on the strait piece of the Co-based amorphous ribbon of $8 \mathrm{~cm}$ in length. There is external magnetic field on the axis $X$. The values of internal magnetic field are lower by the values of demagnetizing field.

The magnetization states corresponding to the observed domain pattern are marked by letters from (a) to (g) in the hysteresis loop depicted in Fig. 5 (domain struc- ture in Fig. 4h has no point on the hysteresis loop). The hysteresis loop was measured on the ribbon of the same length as was used for domain structure observation, in order to simulate the conditions during the observation. The slope of the hysteresis loop is lower compared to the one in Fig. 2 due to the outer demagnetizing field, lowering significantly the internal magnetic field. The hysteresis loop was measured by point-by-point method with commutative steps (in this case the demagnetizing field causes easily creation of the domains with opposite oriented magnetization vector and the hysteresis loop has usual shape without the defect as that in Fig. 1 [5]). In Fig. 5 there are values of external magnetic field on the axis $X$.

\section{Conclusions}

By investigating of magnetic properties of amorphous Co-Fe-B-Si material Vitrovac(R) 6155 U55 F, produced by Vacuumschmelze GmbH \& Co. KG, we found out that the point-by-point methods (both with commutative or summing steps) of the DC hysteresis loops measurement of the long ribbon wound as a core of the toroidal transformer with primary magnetization coil and secondary pick-up coil lead to the incorrect results. It is due to the fact that the magnetization reversal process is performed mainly by domain wall displacement of low number of domain walls, confirmed by the observation of domain pattern during magnetization reversal process. These domain walls at constant magnetic field create energy well by structural rearrangements of the atoms inside the domain wall which acts as pinning centre for the domain wall displacement and to overtake this barrier the additional energy is required. Correct method for measuring of quasi DC hysteresis loop is the continuous method at very low frequency $\mathrm{AC}$ magnetic field $(7 \mathrm{mHz})$.

\section{Acknowledgments}

This work was supported by the Slovak Research and Development Agency under the contract No. APVV-150115 MACOMA and by the Scientific Grant Agency of the Ministry of Education of Slovak Republic and the Slovak Academy of Science, project No. VEGA 1/0330/15 and No. VEGA 1/0377/16. Special thanks to Ing. Milan Vitovský and Dr. Christian Polak, Vacuumschmelze GmbH \& Co. KG Germany for Vitrovac@6155 U55 F.

\section{References}

[1] H. Kronmüller, S. Parkin, Handbook of Magnetism and Advanced Magnetic Materials, Wiley, New York 2007.

[2] Vacuumschmelze, Vitrovac Properties, Fcharacteristics, data sheet.

[3] F. Fiorillo, Measurement and Characterization of Magnetic Materials, Elsevier, Amsterdam 2004.

[4] R. Andrejco, P. Vojtanik, J. Magn. Magn. Mater. 280, 108 (2004).

[5] E. Kneller, Ferromagnetismus, Springer, Berlin 1962. 